\title{
Correction to: Holographic Imaging and Stimulation of Neural Circuits
}

Weijian Yang and Rafael Yuste

\section{Correction to:}

Chapter 43 in: H. Yawo et al. (eds.), Optogenetics, Advances in Experimental Medicine and Biology 1293, https://doi.org/10.1007/978-981-15-8763-4_43

This chapter was inadvertently published with incorrect information in Table 43.1, which has been corrected as below: 
Table 43.1 Comparison between different light illumination approaches used in optical microscopes

\begin{tabular}{|c|c|c|c|c|c|}
\hline & & & $\begin{array}{l}\text { Wide-field } \\
\text { illumination }\end{array}$ & $\begin{array}{l}\text { Point scanning } \\
\text { illumination }\end{array}$ & Holographic illumination \\
\hline \multirow[t]{6}{*}{ Imaging } & \multirow[t]{3}{*}{$\begin{array}{l}\text { Transparent } \\
\text { sample }\end{array}$} & Technique & $\begin{array}{l}\text { Epifluorescence (one } \\
\text { photon) }\end{array}$ & $\begin{array}{l}\text { Confocal } \\
\text { (one photon) }\end{array}$ & Two-photon \\
\hline & & Advantage & $\begin{array}{l}\text { High temporal } \\
\text { resolution }\end{array}$ & $\begin{array}{l}\text { High spatial } \\
\text { resolution }\end{array}$ & $\begin{array}{l}\text { Low background; high } \\
\text { spatial resolution; improved } \\
\text { temporal resolution }\end{array}$ \\
\hline & & Limitation & $\begin{array}{l}\text { Low axial resolution; } \\
\text { phototoxicity }\end{array}$ & $\begin{array}{l}\text { Reduced } \\
\text { temporal } \\
\text { resolution; } \\
\text { phototoxicity }\end{array}$ & \\
\hline & \multirow[t]{3}{*}{$\begin{array}{l}\text { Scattering } \\
\text { sample }\end{array}$} & Technique & $\begin{array}{l}\text { Epifluorescence (one } \\
\text { photon), mainly for } \\
\text { superficial layers }\end{array}$ & Two photon & Two photon \\
\hline & & Advantage & $\begin{array}{l}\text { High temporal } \\
\text { resolution }\end{array}$ & $\begin{array}{l}\text { Low background; } \\
\text { high spatial } \\
\text { resolution }\end{array}$ & $\begin{array}{l}\text { Low background; high } \\
\text { spatial resolution; improved } \\
\text { temporal resolution }\end{array}$ \\
\hline & & Limitation & $\begin{array}{l}\text { High background; } \\
\text { low } \\
\text { spatial resolution; } \\
\text { phototoxicity }\end{array}$ & $\begin{array}{l}\text { Low temporal } \\
\text { resolution }\end{array}$ & \\
\hline \multirow[t]{6}{*}{$\begin{array}{l}\text { Photo } \\
\text { stimulation }\end{array}$} & \multirow[t]{3}{*}{$\begin{array}{l}\text { Transparent } \\
\text { sample }\end{array}$} & Technique & One photon & $\begin{array}{l}\text { One photon/two } \\
\text { photon }\end{array}$ & One photon/two photon \\
\hline & & Advantage & $\begin{array}{l}\text { High temporal } \\
\text { specificity }\end{array}$ & $\begin{array}{l}\text { Improved/high } \\
\text { spatial specificity }\end{array}$ & $\begin{array}{l}\text { Improved/high spatial } \\
\text { specificity; high temporal } \\
\text { specificity }\end{array}$ \\
\hline & & Limitation & $\begin{array}{l}\text { Low spatial } \\
\text { specificity }\end{array}$ & $\begin{array}{l}\text { Low temporal } \\
\text { specificity }\end{array}$ & \\
\hline & \multirow{3}{*}{$\begin{array}{l}\text { Scattering } \\
\text { sample }\end{array}$} & Technique & One photon & Two photon & Two photon \\
\hline & & Advantage & $\begin{array}{l}\text { High temporal } \\
\text { specificity }\end{array}$ & $\begin{array}{l}\text { High spatial } \\
\text { specificity }\end{array}$ & $\begin{array}{l}\text { High spatial specificity; high } \\
\text { temporal specificity }\end{array}$ \\
\hline & & Limitation & $\begin{array}{l}\text { Low spatial } \\
\text { specificity }\end{array}$ & $\begin{array}{l}\text { Low temporal } \\
\text { specificity }\end{array}$ & \\
\hline
\end{tabular}

